\title{
Selective carbon self-wiring from a graphite rod in water under a DC electric field
}

\author{
Hideyuki Sugioka, ${ }^{*}$ Masato Ishikawa, and Taiki Kado \\ Department of Mechanical Systems Engineering, \\ Shinshu University 4-17-1 Wakasato, Nagano 380-8553, Japan
}

(Dated: December 19, 2019)

\begin{abstract}
Selective carbon self-wiring is attractive for the miniaturization of photonic and electrical circuits. In this study, we report that a micro carbon wire grows in the direction opposite to the electric field selectively from a graphite rod in water between parallel electrodes under a DC electric field. Surprisingly, this self-assembled method does not require any preparation for the suspension of carbon materials and it can control the growing position and direction at the same time. Furthermore, we find that the wiring growth rate is approximately proportional to the square of the applied electric field, whereas the wiring phenomenon is not observed under an AC electric field. In addition, the wire grows not from a carbonaceous material but from a graphitic material. Based on these results, we propose that our self-wiring phenomenon occurs owing to the interlayer coupling through the positive (metal) ions in the electric double layer at the growth point, resulting from an induced charge electro-kinetic phenomenon and chemical reactions. We believe that our findings contribute greatly to a new class of microwires.

PACS numbers: 47.57.-s, 85.90.+h, 82.45.Hk, 83.50.Lh
\end{abstract}

\footnotetext{
*hsugioka@shinshu-u.ac.jp
} 


\section{INTRODUCTION}

Self-wiring phenomenon is important as a promising bottom-up fabrication method that does not use top-down photolithography technology for realizing the miniaturization of photonic and electrical circuits [1], which consist of the 3D wiring [2], nanowire (NW) field-effect transistor (FET), crossed NW photonic device [3], NW bio/chemical sensors [4], NW thermogalvanic cell [5], etc. Thus, much effort has been devoted to the wiring phenomenon from the various viewpoints; e.g., fabrication methods, self-organization phenomena, functional materials, applications, etc. In particular, the NWs of carbon materials have been attracted much attention so far since they may have excellent electrical and mechanical characteristics. For example, Chen et al. reported that single-wall carbon nanotubes (SWCNTs) were aligned by an external electric field in ethanol solution [6]. Hermanson et al. [2] reported that microwires having a self-repairing function can be formed simply and predictably by the use of dielectrophoresis (DEP). Zhang et al. demonstrated electric-field-directed growth of SWNTs in chemical-vapor deposition process [7]. Wang et al. demonstrated a controlled assembly of ZnO NWs using DEP [8]. García-Sánchez et al. reported the reversible selfassembly of metal NWs in aqueous suspensions induced by AC electric fields [9]. However, although the wiring phenomena in the suspension of wiring materials have been reported so far, the method that does not use the suspension of wiring materials has not been reported yet. Furthermore, although symmetrical wiring phenomena between electrodes have been reported so far, there is no report for an asymmetrical selective self-wiring phenomenon. In other words, no one reports that a carbon wire can grow in the specific direction from the specific object in water between electrodes. In addition, although wiring phenomena using an induced-charge electro-osmosis (ICEO) and/or DEP, which are subject to an AC electric field, are known well $[2,8,9]$, asymmetrical wiring phenomena using ICEO with a chemical reaction under a DC electric field have not been known yet. In particular, asymmetrical carbon wiring phenomena using the mechanisms have not been explored yet. Therefore, in this study, we report that a micro carbon wire grows in the direction opposite to the electric field selectively from a graphite rod in water between parallel electrodes under a DC electric field. Surprisingly, this self-assembled method does not require any preparation for

the suspension of carbon materials (e.g., graphene oxide, carbon nanotube, etc.) and it can control the growing position and direction at the same time. 


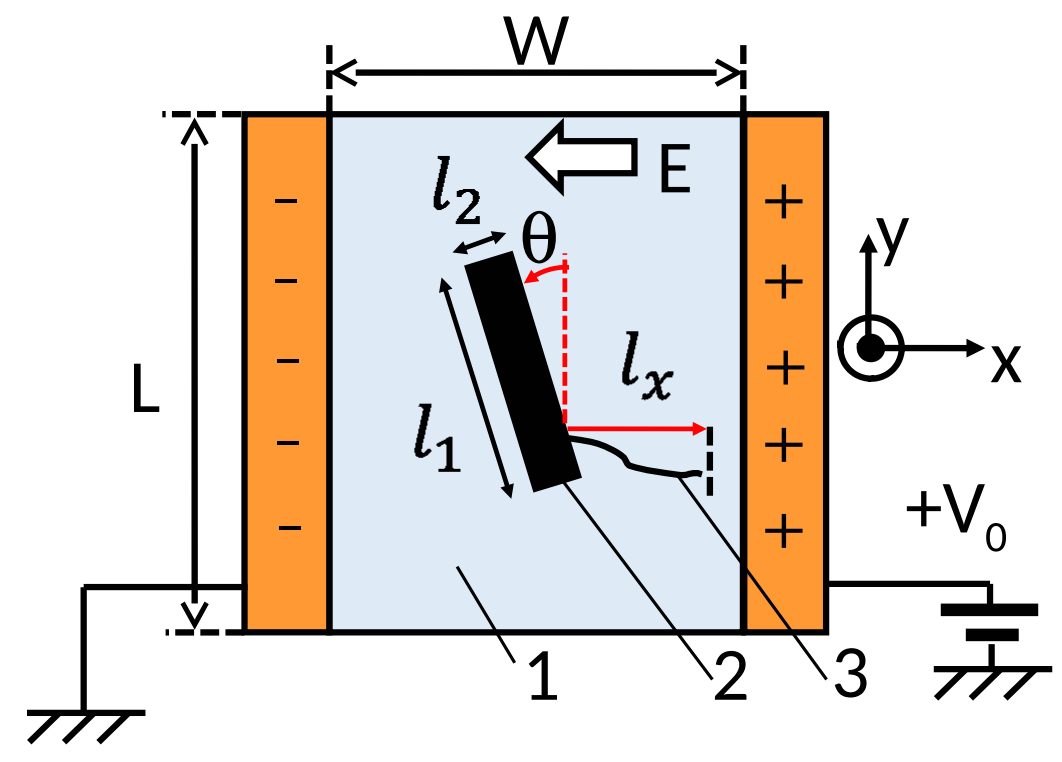

FIG. 1. (Color online) Experimental setup of the selective carbon self-wiring on a graphite rod under a DC electric field. 1: deionized water, 2: graphite rod, and 3: carbon wire. Here, $W=4$ $\mathrm{mm}$ and $L=10 \mathrm{~mm}$.

\section{METHOD}

Figure 1 shows a schematic of the observation system used to investigate our selective carbon self-wiring phenomenon from a graphite rod under a DC electric field. As shown in Fig. 1, a graphite rod of length $l_{1}(=2.0 \mathrm{~mm})$ and diameter $l_{2}(=0.3 \mathrm{~mm})$ was immersed in deionized water (milli-Q, $18.2 \mathrm{M} \Omega \mathrm{cm}$ ) between parallel $(\mathrm{Cu})$ electrodes in a chamber of length $L^{\prime}(=18.4 \mathrm{~mm})$, width $W^{\prime}(=5.5 \mathrm{~mm})$, and depth $d^{\prime}(=9.2 \mathrm{~mm})$. Then, by applying a DC electric voltage $V_{0}$ between the electrodes, we observed the self-wiring phenomenon without using any carbon suspension. After that, we determined the length $l_{x}$ in the $x$ direction at time $t$ by using video data of size $1280 \times 720$ with a frame rate of 30 fps. Further, we repeated such measurement $N_{f}$ times (typically, $N_{f}=3$ or 4 ) at $V_{0}$ using the newly replaced $\mathrm{Cu}$ electrodes with new water, after washing the chamber well with water. In detail, we used the graphite rod (Pilot Co., neox graphite $0.32 \mathrm{~B}$ ) that is produced as a core of a mechanical pencil and considered to be a polycrystalline substance of graphite. Further, the chamber was fabricated from polylactic acid (PLA) using a 3D printer. Specifically, we 


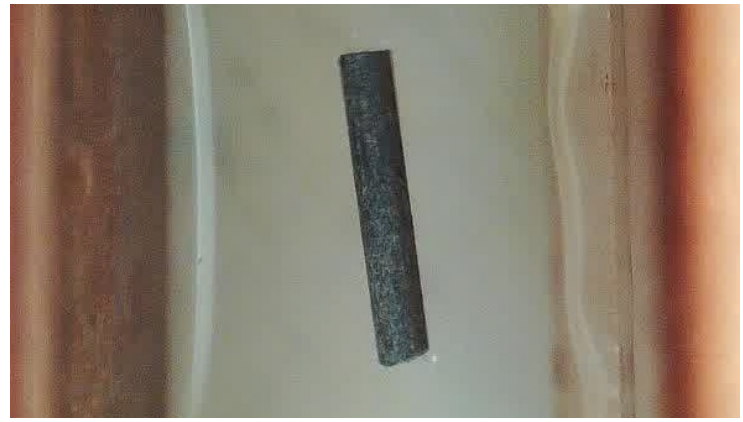

(a) $t=0 \mathrm{~s}$

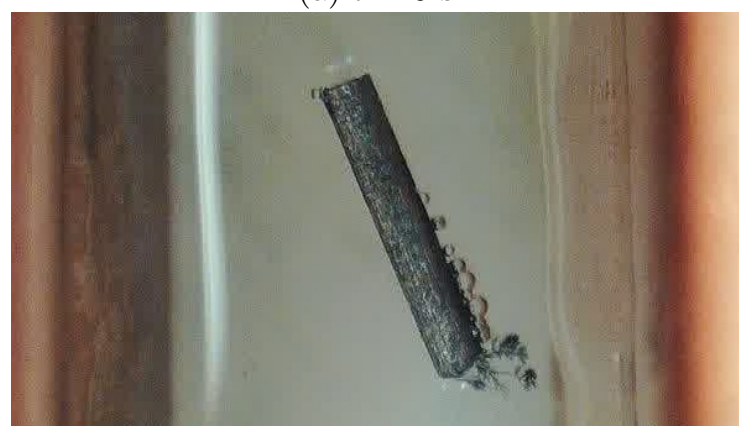

(c) $t=26 \mathrm{~s}$

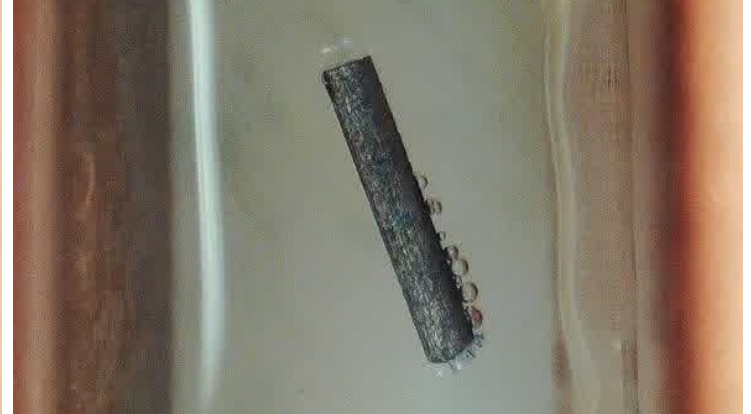

(b) $t=16 \mathrm{~s}$

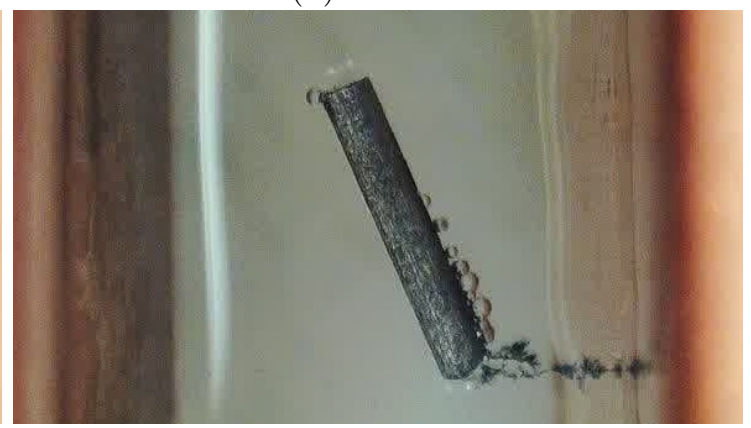

(d) $t=30 \mathrm{~S}$

FIG. 2. (Color online) Photographs of the typical self-wiring process under a DC electric field.

Here, the right electrode is an anode and the left electrode is a cathode; $W=4 \mathrm{~mm}, V_{0}=30 \mathrm{~V}$, $l_{1}=2 \mathrm{~mm}$, and $l_{2}=0.3 \mathrm{~mm}$.

equipped the chamber with the parallel electrodes. Here, the gap distance between the electrodes is $W$ (=4 to $5 \mathrm{~mm}$ ), the length of the electrode is $L(=10 \mathrm{~mm})$, and the depth of the chamber is $d$ (=7 to $9 \mathrm{~mm})$. Furthermore, we connected a resistance of $1 \mathrm{k} \Omega$ to protect a circuit, although the effect was usually negligible. Note that we usually applied an electric voltage only while a wire was growing from a graphite rod to the right positive electrode (anode).

\section{RESULTS}

\section{A. Self-wiring process}

Figures 2(a), 2(b), 2(c), and 2(d) show the photographs of the typical self-wiring process at $t=0,18,26$, and $30 \mathrm{~s}$, respectively under the existence of a dc electric field. As shown in Figs. 2(b) and 2(c), the carbon wire started to grow at $t=t_{1}(=13 \mathrm{~s})$ from the right side 
surface of the graphite rod and grew in the opposite direction of the electric field. Then, it was connected to the right electrode (anode) at $t=t_{2}(=30 \mathrm{~s})$ as shown in Fig. 2(d). Although the carbon wire started to grow at the edge of the rod in Fig. 2, it was not common; i.e., the carbon growth generally started from the right side surface (of the graphite rod), which has an electric double layer consisting of the negative charge induced on a graphite surface and positive ions in water. Furthermore, we did not observe the growth of carbon materials from the left negative electrode (cathode) at least during this self-wiring process except one exceptional case, which probably resulted from the insufficient cleaning of the chamber. In this sense, we consider that the wiring phenomenon occurred selectively from the graphite rod. However, we could observe that wires grew from the cathode after the wire grew from the graphite to the anode. Therefore, the selectivity comes from the large difference of the wire growth rate between the wiring from the graphite and the wiring from the cathode. Furthermore, as shown in Fig. 2, the graphite rod rotated and tried to align in the direction of the electric field by the effects of ICEO and DEP torques. However, because of the existence of the meniscus between the electrodes, the rod was stopped at $\theta=\theta_{s}$ (e.g., $\left|\theta_{s}\right| \simeq 2$ to $\left.34^{\circ}\right)$ without aligning in the direction of the electric field. Thus, the wiring phenomenon occurred under the existence of ICEO and DEP phenomena.

\section{B. Characteristics of self-wiring}

Figures 3(a) and 3(b) show the dependence of $l_{x}$ on $t$ at $V_{0}=30$ and $40 \mathrm{~V}$, respectively. Here, three independent measurements $\left(N_{f}=3\right)$ were done at the same applied voltage for the different new electrodes with a well-washed chamber. As shown in Figs. 3(a) and $3(\mathrm{~b})$. we find a large deviation in $t_{1}, \Delta t\left(\equiv t_{2}-t_{1}\right)$, and the average growth rate $V_{\text {ave }}$ [三 $\left.\left(l_{x}\left(t_{2}\right)-l_{x}\left(t_{1}\right)\right) / \Delta t\right]$ during a wire growing period. Probably, the initial wiring process starts as a stochastic event that a graphite cluster (cleaved from the graphite rod and flowing in chamber owing to ICEO flows) approaches again to the right side of the graphite rod. Thus, there is a large deviation in $t_{1}$. Furthermore, although the wire grows selectively from the graphite, the position is only limited to the relatively wide region of the right side of the rod. Thus, the deviation in $\Delta t$ is also large since the local electric field and the supply of the carbon materials depend on the growing position. Figure 3(c) shows the dependence of $V_{\text {ave }}$ on $V_{0}$. In Fig. 3(c), the circles show the average over 3 or 4 independent measurements 
$\left(N_{f}=3\right.$ to 4$)$ and the error bars show the standard deviation for the measurements. From Fig. 3(c), although there is a large deviation, we find that our self-wiring phenomenon has a nonlinear characteristics described by $V_{a v e} \propto V_{0}^{2}$. In other words, $V_{\text {ave }}$ is proportional to the ICEO flow velocity $U_{I C E O}$ because $U_{I C E O}$ is proportional to the square of $V_{0}$. Probably, the carbon materials are supplied by the ICEO flow around the carbon rod, although they are not used for the wiring from the electrodes and the wiring from the left side region of the carbon rod. Furthermore, Fig. 3(d) shows the dependence of $1 / t_{1}$ on $V_{0}$. Here, $1 / t_{1}$ shows how many seeds can be produced stochastically in 1 s. In Fig. 3(d), the circles show the individual data, whereas the vertical lines show the region between the maximum and minimum data at each $V_{0}$. From Fig. $3(\mathrm{~d})$, we find that the maximum value of $1 / t_{1}$ decreases monotonically at $V_{0}>30 \mathrm{~V}$, whereas it increases at $V_{0}<30 \mathrm{~V}$. Probably, the decreasing

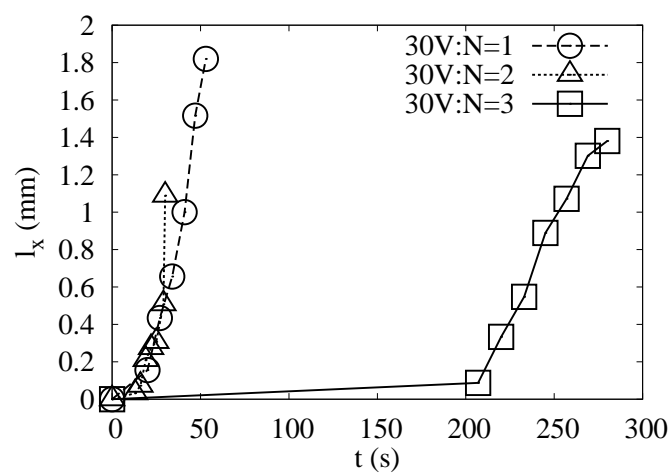

(a) Dependence of $l_{x}$ on $t$ at $V_{0}=30 \mathrm{~V}$

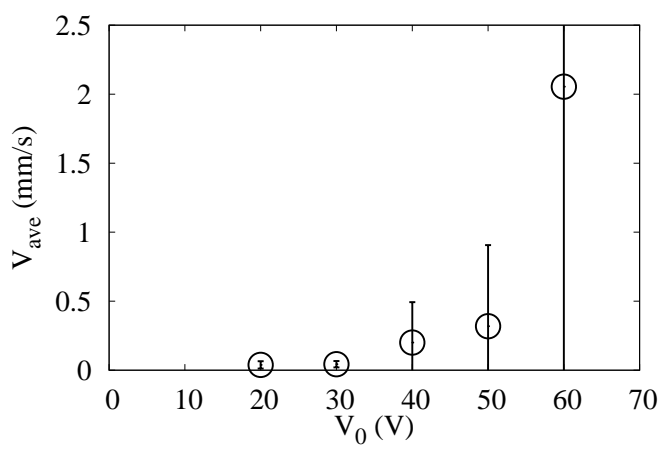

(c) Dependence of $V_{\text {ave }}$ on $V_{0}\left(N_{f}=3\right.$ to 4$)$

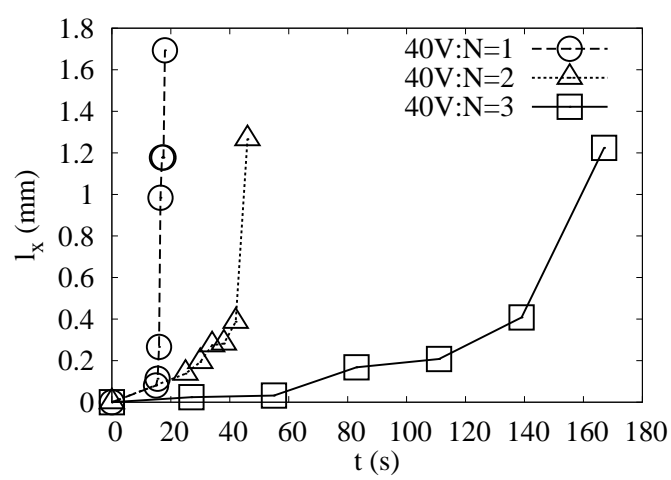

(b) Dependence of $l_{x}$ on $t$ at $V_{0}=40 \mathrm{~V}$

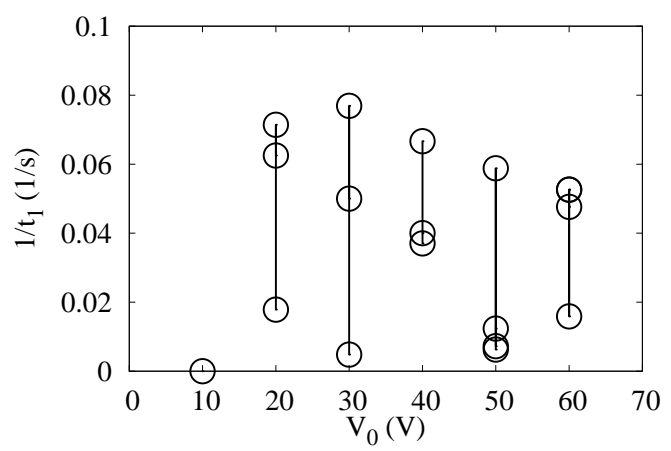

(d) Dependence of $1 / t_{1}$ on $V_{0}$

FIG. 3. Characteristics of the selective carbon self-wiring process under a DC electric field. Here, $W=4 \mathrm{~mm}, l_{1}=2 \mathrm{~mm}$, and $l_{2}=0.3 \mathrm{~mm}$. 


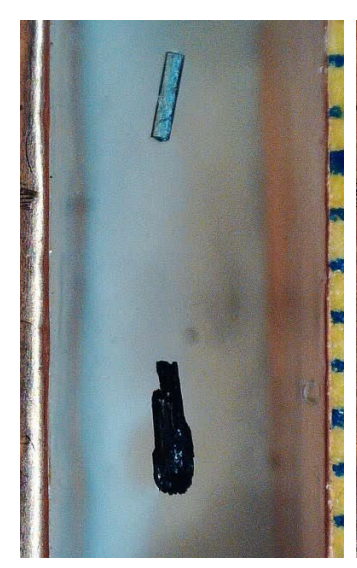

(a) $t=0 \mathrm{~s}$

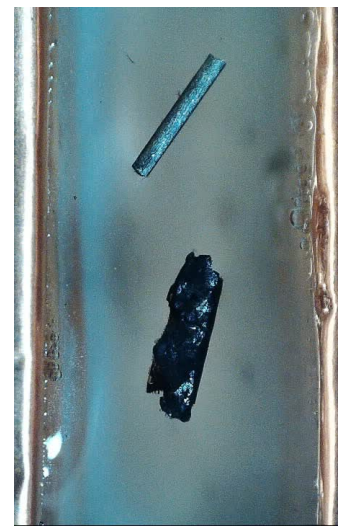

(d) $t=0 \mathrm{~s}$

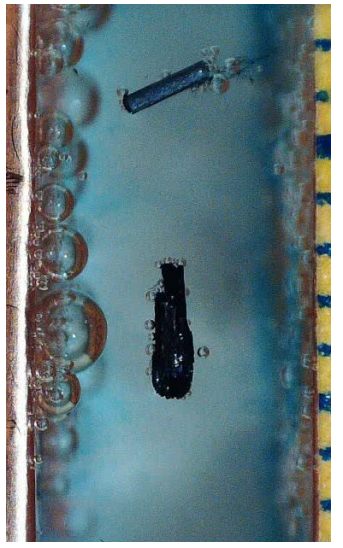

(b) $t=182 \mathrm{~s}$

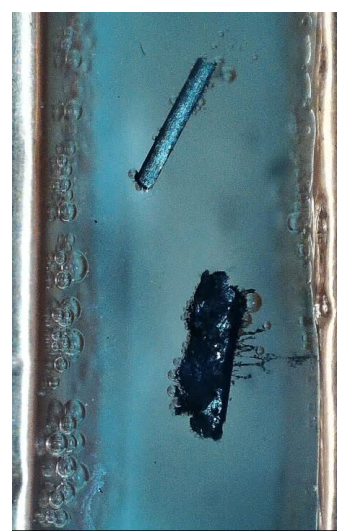

(e) $t=38 \mathrm{~s}$

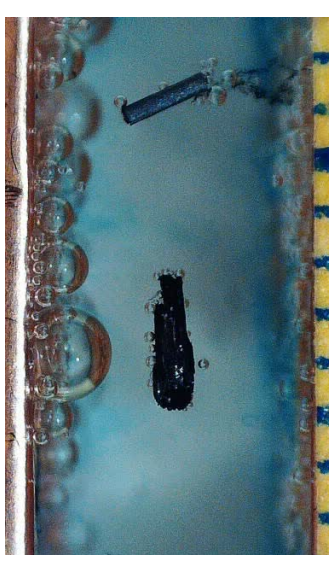

(c) $t=202 \mathrm{~s}$

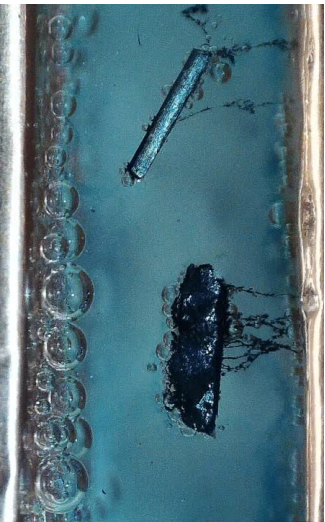

(f) $t=115 \mathrm{~s}$

FIG. 4. (Color online) The effects of the rod materials under a DC electric field. In (a), (b), and (c), the comparison between Material 1 (graphite; the upper rod; $0.3 \times 1.7 \mathrm{~mm}^{2}$ ) and Material 2 (charcoal; the lower rod; $0.7 \times 2.6 \mathrm{~mm}^{2}$ ) was done, whereas the comparison between Material 1 (graphite; the upper rod; $0.3 \times 2.9 \mathrm{~mm}^{2}$ ) and Material 3 (active charcoal; the lower rod; $0.9 \times 3.9$ $\mathrm{mm}^{2}$ ) was done in (d), (e), and (f). Here, the left electrode is a cathode, whereas the right electrode is an anode; $W=5 \mathrm{~mm}$ and $V_{0}=30 \mathrm{~V}$.

at $V_{0}>30 \mathrm{~V}$ results from the increasing of bubbles due to the chemical reactions at the rod and electrodes, whereas the increasing at $V_{0}<30 \mathrm{~V}$ results from the increasing of the carbon supply due to the increasing of an ICEO flow velocity.

\section{Effect of rod materials}

To clarify the mechanism of this self-wiring phenomenon, we compared three kinds of carbon materials. Namely, graphite (Material 1: Pilot Co.), charcoal (Material 2: Comeri 
Co.), and active charcoal made from palm trees (Material 3: Gex Co. ; super-adsorbed activated carbon). Here, Materials 1 and 3 are classified non-porous and porous graphitic materials, respectively, whereas Material 2 is classified non-porous carbonaceous material. Figures 4(a), 4(b), and 4(c) show the comparison of wiring process between Materials 1 and 2, whereas Figs. 4(d), 4(e), and 4(f) show the comparison of wiring process between Materials 1 and 3. As shown in Fig. 4, we could not observe the wiring phenomenon for Material 2 at least during 4 min, whereas we observed the wiring phenomenon for Materials 1 and 3. Thus, we consider that graphitic carbon material is required for the selective carbon self-wiring phenomenon. Furthermore, by the following measurements, we find that this wiring phenomenon occurs only under a DC electric field. That is, we put a graphite rod between the electrodes of $W=5 \mathrm{~mm}$ and applied an AC voltage (which peak voltage is $32 \mathrm{~V}$ ) continuously at frequencies $f=1,10,100,1000$, and $10000 \mathrm{~Hz}$. Then, we applied an DC voltage $\left(V_{0}=30\right)$. Further, we repeated this measurement 3 times. By this experiment, the wiring phenomenon was never observed at least for 5 min under AC electric fields, whereas the wiring phenomenon was observed every time under a DC electric field. Thus, we consider that this selective carbon self-wiring phenomenon occurs only under DC electric fields, although it is relevant to ICEO phenomena.

\section{Structures of wires}

To observe the surface of the wires with a scanning electron microscope (SEM), we prepared two samples (Samples 2 and 3) with two different methods. That is, after setting a pair of $\mathrm{Cu}$ tapes of thickness $t^{\prime} \simeq 0.07 \mathrm{~mm}$ on a glass substrate as parallel electrodes of the distance $W \simeq 3 \mathrm{~mm}$, we placed a carbon rod and a water droplet between the electrodes. Then, by applying $V_{0}=30 \mathrm{~V}$, we obtained Sample 2. As for Sample 3, we performed the same procedure with that of Sample 2. However, after obtaining a wire between the graphite rod and the anode, we removed the wire and the graphite rod from the droplet and we applied $V_{0}=30 \mathrm{~V}$ again. Then, we obtained Sample 3. Figures 5(a) and 5(b) show the photographs of Samples 2 and 3, respectively, whereas Figs. 5(c) and 5(d) show the SEM pictures of Samples 2 and 3, respectively. From Figs. 5(c) and 5(d), we find that the carbon wires obtained by our method have a fine network structure. Furthermore, since we could also obtain carbon wires even from Sample 3, we can recognize that the suspension of car- 


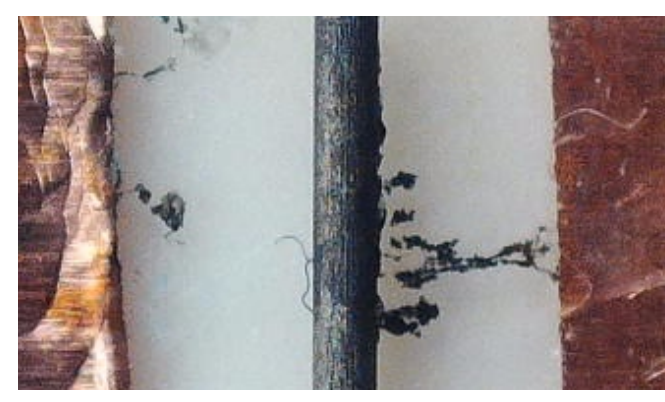

(a) Photograph of Sample 2

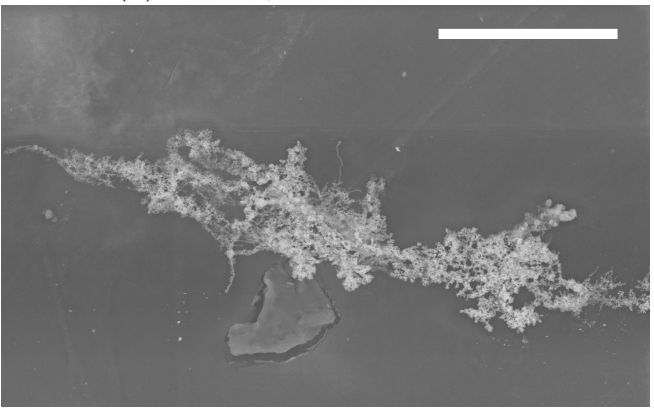

(c) SEM picture of the wire in Sample 2

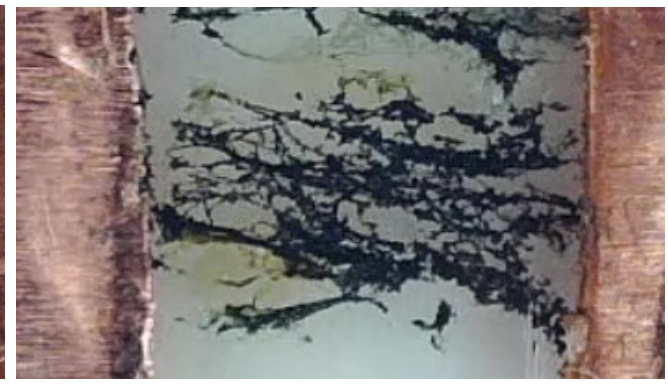

(b) Photograph of Sample 3

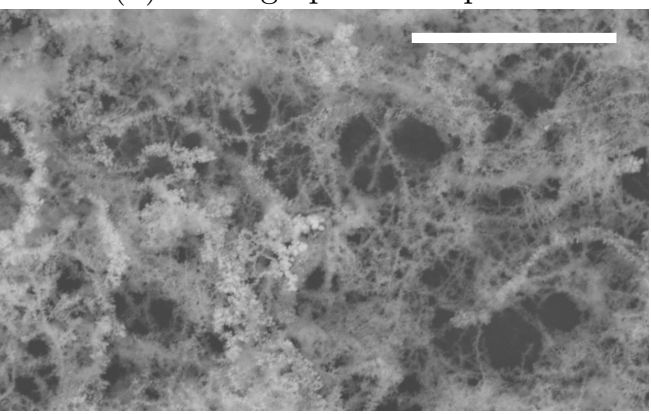

(d) SEM picture of the wire in Sample 3

FIG. 5. (Color online) Structures of wires. Here, $W=3 \mathrm{~mm}$ and $V_{0}=30 \mathrm{~V}$; the scale bar in (a) shows $50 \mu \mathrm{m}$; the scale bar in (b) shows $10 \mu \mathrm{m}$.

bon materials is naturally prepared during our self-wiring process. In addition, this result supports that the wire is formed selectively on the graphite rod owing to the difference of the wire growth rate between the graphite surface and the $\mathrm{Cu}$ electrode surface, although we already discussed this issue in Sec. III-A.

\section{E. Composition analysis}

Figure 6 shows the SEM pictures of measured areas for the composition analysis. Here, Sample 1 was prepared by the method in Sec. II. As shown in Fig. 6(a), the surface of the positive electrode (in Sample 1) is relatively flat, whereas the surface of the negative electrode (in Sample 1) has seeds for wires, as shown in Fig. 6(b). This is because the dissolution of $\mathrm{Cu}$ occurs on the positive electrode (anode), whereas the deposition of $\mathrm{Cu}$ occurs on the negative electrode (cathode) owing to the chemical reactions. Furthermore, in Fig. 6(c) (for Sample 2), we can recognize the white wire areas on the glass substrate and the white stains (which are considered to be seeds of wires) on the graphite surface, whereas in Fig. 6(d) (for Sample 3), we find a network structure similar to that of Fig. 5(d). 

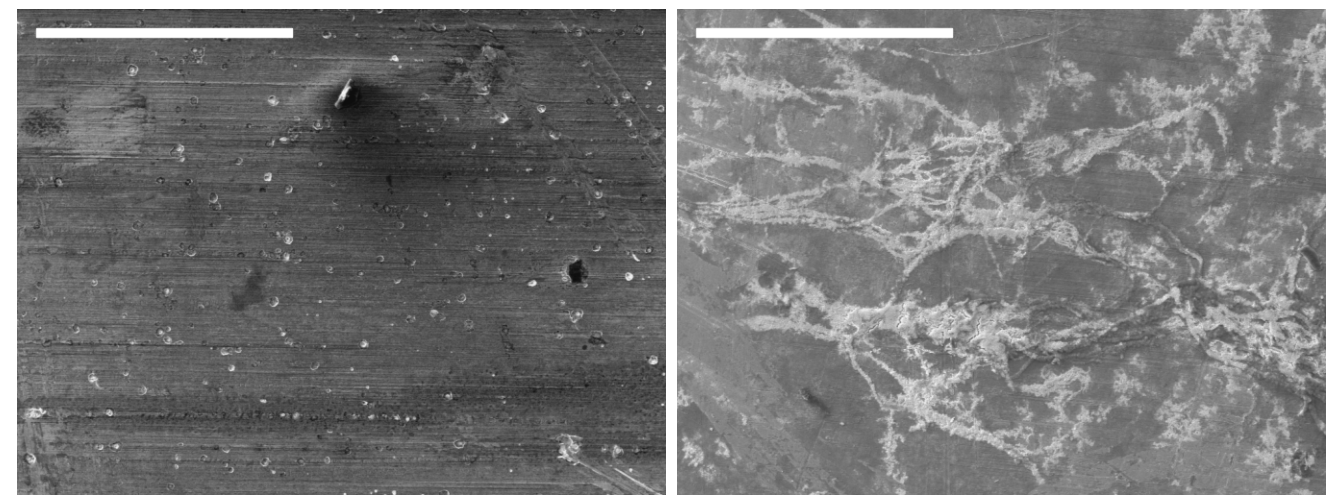

(a) SEM picture of the anode in Sample 1

(b) SEM picture of the cathode in Sample

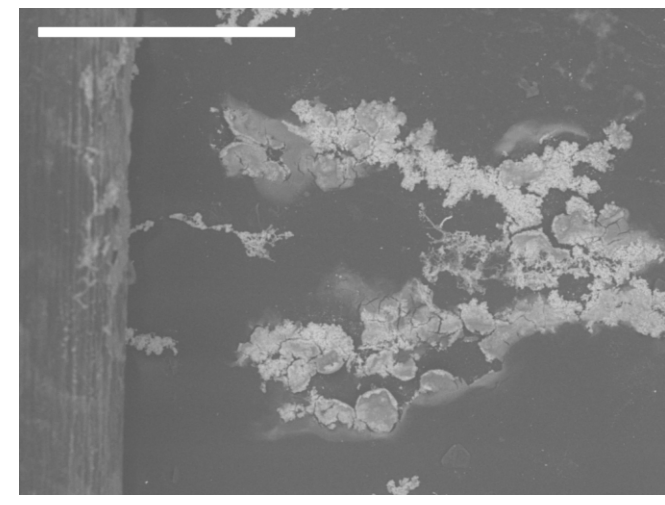

(c) SEM picture of the graphite rod and

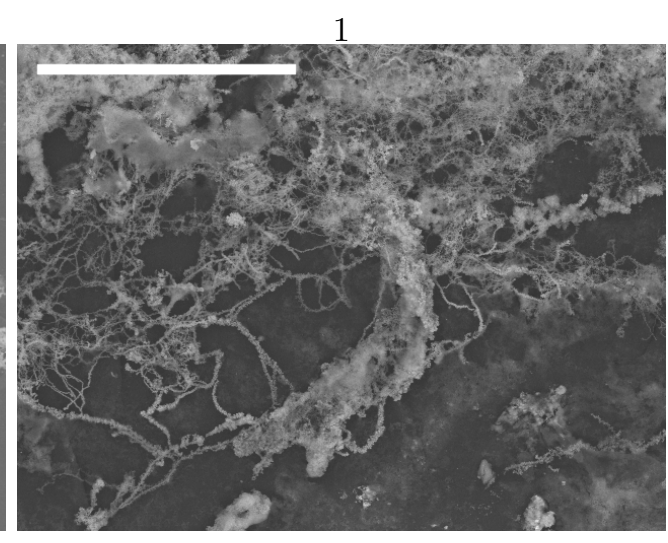

(d) SEM picture of the wire in Sample 3

the wire in Sample 2

FIG. 6. SEM pictures for the composition analysis. In (a) to (c), the scale bars show $250 \mu \mathrm{m}$. In (d), the scale bar shows $50 \mu \mathrm{m}$. Here, $W \simeq 3 \mathrm{~mm}$ and $V_{0}=30 \mathrm{~V}$.

Table 1 shows the results of the composition analysis by the energy dispersive X-ray spectrometry (EDS). Firstly, we find that carbon materials deposit on the both positive and negative electrodes. This is probably because, although the surface of the carbon materials has negative charges intrinsically, positively charged carbon clusters exist by the adsorption of the excess metal ions. Secondly, the white stain on a graphite rod in Sample 2 contains copper of 3.6 to 11.2 atom\%. Thus, the white stain is considered to be the seed of the wire due to the deposition of the copper. Thirdly, the wires in Samples 1 to 3 consist of carbon (20.5 to 70.7 atom\%), copper (8.8 to 75 atom\%), and oxygen (4.6 to 34.1 atom\%). Therefore, the wire is considered to be an intercalational compound, as discussed later (in Sec. V). In particular, the percentage of the carbon content in the wire of Sample 2 (66.4 to 70.7 atom\%) is the highest, followed in order by that of Sample 3 (41.9 to 62.8 atom\%) and 
TABLE I. Results of the composition analysis

\begin{tabular}{|l|l|l|l|l|}
\hline Sample & measured position & $\mathrm{C}($ atom\%) & $\mathrm{Cu}$ (atom\%) & $\mathrm{O}$ (atom\%) \\
\hline \hline Sample 1 & positive electrode & $50.9-56.1$ & $43.9-49.1$ & 0.0 \\
\hline Sample 1 & negative electrode & $49.0-75.4$ & $6.4-47.1$ & $2.5-17.3$ \\
\hline Sample 1 & wire on a negative electrode & $20.5-39.1$ & $43.9-75.0$ & $4.6-14.0$ \\
\hline Sample 2 & graphite & 98.5 & 0.9 & 0.0 \\
\hline Sample 2 & white stain on graphite & $65.9-75.4$ & $3.6-11.2$ & $17.3-21.7$ \\
\hline Sample 2 & wire & $66.4-70.7$ & $8.8-13.4$ & $11.9-22.6$ \\
\hline Sample 3 & wire & $41.9-62.8$ & $11.7-23.4$ & $22.6-34.1$ \\
\hline
\end{tabular}

that on a negative electrode of Sample 1 (20.5 to 39.1 atom\%). In other words, we find that the wire that grows from the graphite rod contains more carbon than the wire that grows from the copper electrode.

\section{F. Electrical characteristics}

Figure 7 summarizes the electrical characteristics of the wire. Specifically, Fig. 7(a) shows the dependence of the current $I$ on $t$ during the wiring process under $V_{0}=32 \mathrm{~V}$ and $W=4.3 \mathrm{~mm}$. As shown in Fig. 7(a), $I$ increases rapidly until $t \simeq 4 \mathrm{~s}$ and then approximately keeps the value constant until $t=267 \mathrm{~s}$; i.e., $I \simeq 0.8 \mathrm{~mA}$ at $t<267 \mathrm{~s}$. This is because the concentration of $\mathrm{Cu}$ ions increases rapidly at $t<4 \mathrm{~s}$ owing to the chemical reactions at the electrodes. Then, it becomes constant since the reaction rate becomes constant owing to the formation of the steady electric double layer at the both electrodes. Further, $I$ increases again since the wire starts to grow at $t \geq 267 \mathrm{~s}$. Figure $7(\mathrm{~b})$ shows the dependence of $I$ on $V_{0}$ after the wiring process, whereas Fig. 7(c) shows the dependence of $I$ on $V_{0}$ after we removed the wire. Here, we applied a sinusoidal wave voltage with a frequency $f=100 \mathrm{~Hz}$ and a peak voltage of $\pm 1 \mathrm{~V}$. In Figs. 7(b) and 7(c), the circles show the experimental data, whereas the solid lines show the regression lines determined by the least-square method. From the inclination of Fig. 7(c), we determine the total resistance of the cell as $R_{t}^{\text {wireless }} \simeq 2 R_{1} \simeq 562 \mathrm{k} \Omega$ when there is no wire, where $R_{1} \simeq 281 \mathrm{k} \Omega$ is a resistance between the graphite rod and the electrodes. Here, we assume that the graphite 
rod is placed at the center between the electrodes. Further, we determine the total resistance of the cell as $R_{t}^{\text {wire }} \simeq R_{1}+\frac{R_{1} R_{2}}{R_{1}+R_{2}} \simeq 415 \mathrm{k} \Omega$ when there is a wire, where $R_{2}$ is a resistance of the wire. Thus, we estimate that $R_{2} \simeq 256 \mathrm{k} \Omega$. Figure $7(\mathrm{~d})$ shows the photograph of the cell after the wiring process. Since there are two wires of length $l \sim 1.0 \mathrm{~mm}$ and cross section $A$, we obtain that $R_{t}^{\text {wire }} \sim \rho^{\text {wire }} \frac{l}{2 A}$, where $\rho^{\text {wire }}$ is the resistivity of the wire. Although it is difficult to estimate $A$ because of the network structure, if we estimate that the width $\phi$ of the wire is approximately 0.1 to $1.0 \mu \mathrm{m}$ from Figs. 5(d) and $7(\mathrm{~d})$, we consider that $A \sim \frac{\pi \phi^{2}}{4} \sim 7.85 \times 10^{-15}$ to $7.85 \times 10^{-13}$. Thus, we consider that $\rho^{\text {wire }} \sim R_{2} \frac{2 A}{l} \sim 4.02 \times 10^{-6}$ to $4.02 \times 10^{-4} \Omega \mathrm{m}$. Since the resistibity of graphite is known as $\sim 10^{-5} \Omega \mathrm{m}$, we consider that the estimation is reasonable to some extent.

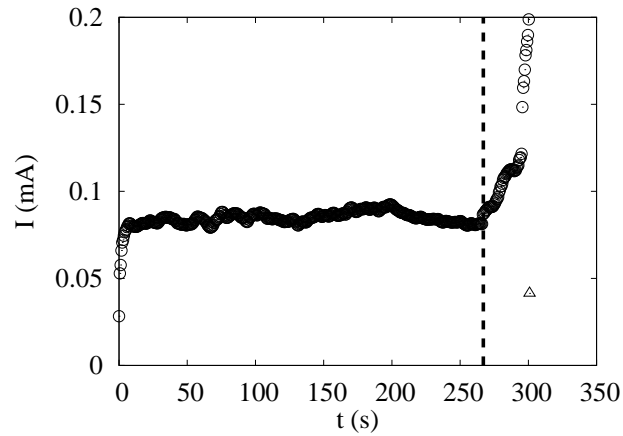

(a) $I$ vs. $t$ during the wiring process

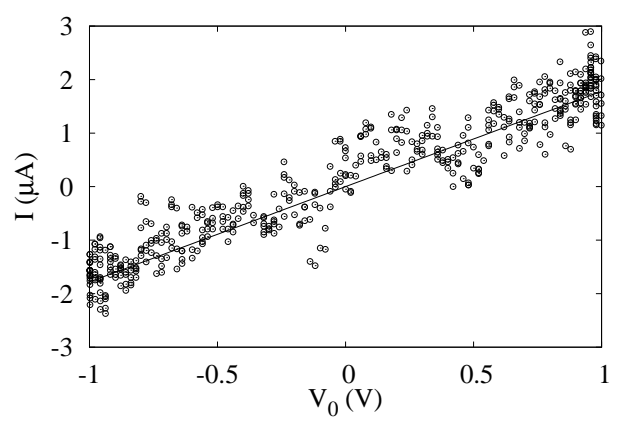

(c) $I$ vs. $V_{0}$ after removing the wire

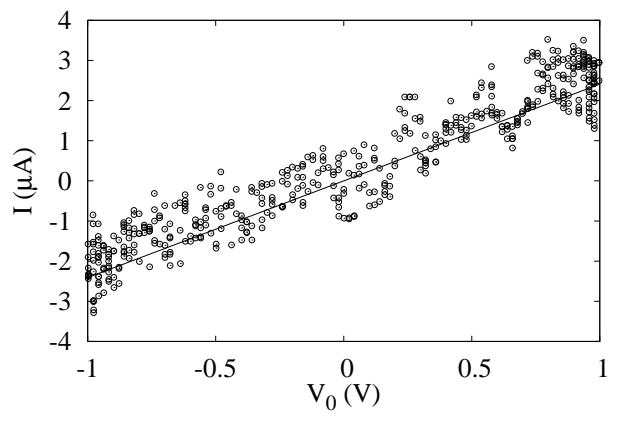

(b) $I$ vs. $V_{0}$ after the wiring process

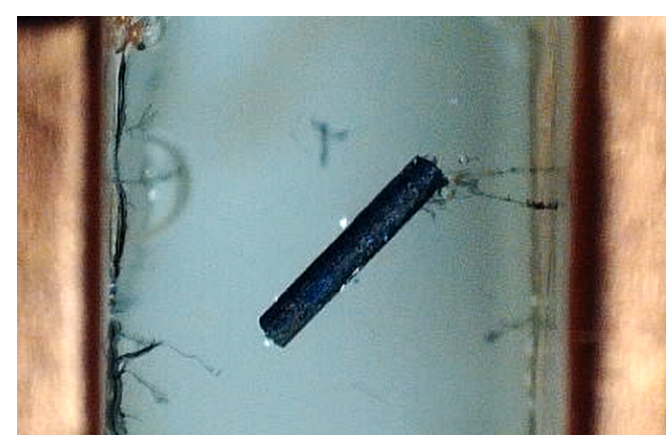

(d) Photograph after the wiring process

FIG. 7. (Color online) Electrical characteristics (for Sample 1). In (a), $V_{0}=32$ V. In (b) and (c), $f=100 \mathrm{~Hz}$. Here, $W=4.3 \mathrm{~mm}$. 


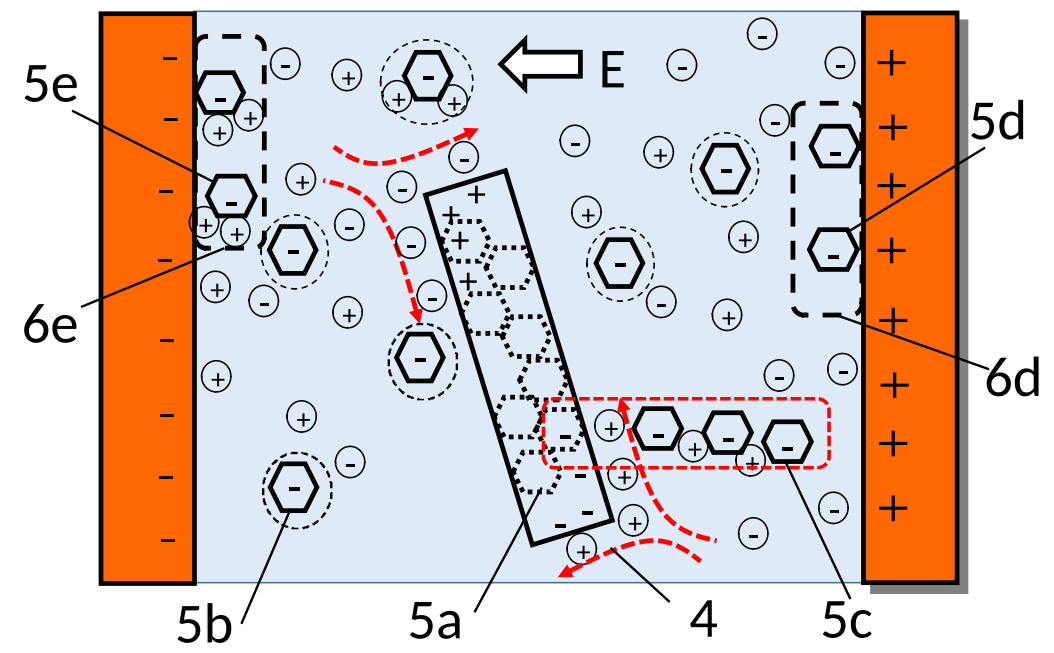

FIG. 8. (Color online) Schematic of the presumed mechanism for the selective carbon self-wiring phenomenon on a graphite rod under a DC electric field. 4: ICEO flow, 5a: graphite in a carbon rod, 5b: carbon (e.g., graphene oxide, graphite, etc.) cluster in a water, 5c: carbon cluster probably connected by the interlayer coupling through intercalated positive (metal) ions in a carbon water, 5d: carbon cluster on a positive electrode due to EPD, 5e: carbon cluster on a negative electrode due to EPD, 6d: carbon cluster layer on a positive electrode due to EPD, and 6e: carbon cluster layer on a negative electrode due to EPD.

\section{DISCUSSION}

In this study, we have first reported that a micro carbon wire grows in the direction opposite to the electric field selectively from a graphite rod in water between parallel electrodes under a DC electric field. Since this self-assembled method does not require any preparation for the suspension of carbon materials, it is convenient as a simple fabrication method for growing a carbon wire. In addition, since it can control the growing position and direction, it is useful to realize various complex NW photonic and electrical circuits. Moreover, we found that the wiring growth rate was approximately proportional to the square of the applied electric field. Thus, our wiring phenomenon is related to the ICEO (or AC electro-osmosis) because the ICEO flow velocity is proportional to the square of the applied 
electric field. However, any wiring phenomena were not observed under an AC electric field in our experiment. Therefore, we consider that our wiring phenomenon occurs by the synergistic effect of the ICEO flows and chemical reactions around the graphite rod. That is, by the synergistic effect, the carbon materials [nano-scale graphene oxide (GO) plates and/or graphite clusters] may be peeled from the graphite rod and the carbon materials can join on the negatively charged surface through the intercalated positive (metal) ions in the electric double layer of the graphite rod (or the tip of the wire) accompanied by ICEO. Figure 8 shows the schematic of this presumed mechanism for the selective self-wiring phenomenon. As shown in Fig. 8, the main mechanism is that the cleaved or peeled carbon materials (5b) (flowing by the ICEO flows) form a connection with the graphite material on a carbon rod (5a) or the edge carbon material (5c) by the interlayer coupling through the intercalated positive (metal) ions in water. This model explains the facts: (1) the wiring phenomenon is observed only on the right cathodic surface of the carbon rod; (2) the wiring phenomenon is not observed on Material 2 (carbonaceous material) but observed on Materials 1 and 3 (graphitic material); and (3) the wiring phenomenon is not observed on the left cathodic electrode at least within $t_{2}$ although it can be observed later by the adhesion of the graphitic material on the left electrode. Furthermore, if graphite clusters [and/or GO plates] are produced by the cleavage process due to ICEO flows, a part of unused graphite clusters [and/or GO plates] will be deposited on the right anodic electrode through electrophoretic deposition (EPD) [10], because it is well known that graphite clusters [and/or GO plates] are negatively charged at $p H \sim 7$, although graphite clusters can be charged positively owing to the excess adsorption of the metal ions. In fact, by the composition analysis in Table 1, we find that carbon materials deposit on the both positive and negative electrodes, as mentioned before.

\section{CONCLUSION}

In conclusion, we have demonstrated selective carbon self-wiring from a graphite rod in water under a DC electric field. Furthermore, the mechanism responsible for the selective carbon self-wiring was identified as the synergistic effect of the ICEO flows and chemical reactions; i.e., we propose a model that the cleaved carbon materials flowing around a carbon rod owing to ICEO form a connection with the graphite material on a carbon rod or the edge carbon material by the interlayer coupling through the intercalated positive (metal) 
ions in water.

[1] Y. Li, F. Qian, J. Xiang, and C. M. Lieber, Materials Today 9, 18 (2006).

[2] K. D. Hermanson, S. O. Lumsdon, J. P. Williams, E. W. Kaler, and O. D. Velev, Science 294, $1082(2001)$.

[3] X. Duan, Y. Huang, Y. Cui, J. Wang, and C. M. Lieber, Nature 409, 66 (2001).

[4] Y. Cui, Q. Wei, H. Park, and C. M. Lieber, Science 293, 1289 (2001).

[5] R. Hu, B. A. Cola, N. Haram, J. N. Barisci, S. Lee, S. Stoughton, G. Wallace, C. Too, M. Thomas, A. Gestos, M. E. d. Cruz, J. P. Ferraris, A. A. Zakhidov, and R. H. Baughman, Nano Letters 10, 838 (2010).

[6] X. Q. Chen, T. Saito, H. Yamada, and K. Matsushige, Applied Physics Letters 78, 3714 (2001).

[7] Y. Zhang, A. Chang, J. Cao, Q. Wang, W. Kim, Y. Li, N. Morris, E. Yenilmez, J. Kong, and H. Dai, Applied Physics Letters 79, 3155 (2001).

[8] D. Wang, R. Zhu, Z. Zhou, and X. Ye, Applied Physics Letters 90, 103110 (2007).

[9] P. García-Sánchez, J. J. Arcenegui, H. Morgan, and A. Ramos, Applied Physics Letters 106, $023110(2015)$.

[10] S. J. An, Y. Zhu, S. H. Lee, M. D. Stoller, T. Emilsson, S. Park, A. Velamakanni, J. An, and R. S. Ruoff, The Journal of Physical Chemistry Letters 1, 1259 (2010). 